Revue d'histoire de l'Amérique française

REVUE D.HISTOIRE DE L'AMÉRIQUE FRANÇAISE

\title{
Inventaire de l’Église canadienne à la fin de 1764
}

\section{Marcel Trudel}

Volume 9, numéro 3, décembre 1955

URI : https://id.erudit.org/iderudit/301722ar

DOI : https://doi.org/10.7202/301722ar

Aller au sommaire du numéro

Éditeur(s)

Institut d'histoire de l'Amérique française

ISSN

0035-2357 (imprimé)

1492-1383 (numérique)

Découvrir la revue

Citer cet article

Trudel, M. (1955). Inventaire de l'Église canadienne à la fin de 1764. Revue

d'histoire de l'Amérique française, 9(3), 313-346.

https://doi.org/10.7202/301722ar d'utilisation que vous pouvez consulter en ligne.

https://apropos.erudit.org/fr/usagers/politique-dutilisation/ 


\title{
INVENTAIRE DE L'ÉGLISE CANADIENNE À LA FIN DE 1764 *
}

\begin{abstract}
SOMMAIRE
$\mathrm{Y}$ eut-il un exode du clergé ? Les nombreux vides causés par les décès. Le tiers des effectifs demeure à peu près perdu. Dénombrement de l'Eglise canadienne à la fin de 1764: Acadie, Gouvernement de Québec, Gouvernement des Trois-Rivières, Gouvernement de Montréal, région des Grands Lacs, région des Illinois. Une grave pénurie de prêtres. Les raisons d'espérer.
\end{abstract}

Le 10 août 1764, les Canadiens s'étaient vu imposer officiellement et définitivement un régime anglais et protestant. L'Église canadienne avait bien des motifs de s'inquiéter de son avenir: le traité de Paris n'a garanti la liberté de religion que d'une façon vague et ambiguë; les catholiques sont exclus, en principe, de toute charge publique s'ils ne consentent à prêter un serment d'abjuration, le Test; les dîmes n'ont aucune existence légale; les Jésuites et les Récollets n'ont point la permission de se recruter; l'Angleterre a consenti à tolérer tout juste un Supérieur du Clergé; toute hiérarchie papale et toute correspondance avec Rome sont officiellement interdites; le gouverneur Murray a tout à fait éliminé Montgolfier comme évêque-élu et même comme grand-vicaire, il a imposé au choix du Chapitre un chanoine timide et pauvre, Jean-Olivier Briand. A l'automne de 1764, ce dernier est en route pour l'Europe où, protégé par Murray, il va tenter de se faire accepter et de se faire consacrer; or le crédit de Murray baisse de plus en plus: le succès de Briand

\footnotetext{
* Extrait d'un ouvrage en deux volumes, à paraître en 1956: L'Eglise canadienne sous le régime militaire, 1759-1764.
} 
restera longtemps douteux. Si Briand échoue, qu'adviendra-t-il de l'Église canadienne?

Il y a encore un motif très grave d'inquiétude: les vides nombreux que les décès et les départs produisent dans le clergé. Au début de 1759, l'Église canadienne comptait 196 prêtres, séculiers et réguliers; à la fin de 1760 , ce nombre est tombé à 163 ; à la fin de 1764, il est réduit à 137.

\section{$Y$ eut-il un exode du clergé?}

On a souvent parlé de l'exode de notre noblesse, de l'exode de nos fonctionnaires et de nos bourgeois: la diminution rapide de notre clergé peut-elle s'expliquer par un exode?

Assurément, il y eut des départs, et en assez grand nombre. Ces départs, nous l'avons démontré dans un chapitre précédent ${ }^{1}$, commencent aussitôt après la capitulation de Québec. Et l'exemple vient tout de suite de haut. Deux chanoines canadiens, Charles-Ange Collet et Gilles-Louis Cugnet, quittent volontairement le pays à l'automne de 1759 , passant en France sur un navire anglais; le chanoine Collet, pour sa part, a la permission du grand-vicaire Briand; son confrère Cugnet est tout jeune, il n'a que 28 ans. Comme l'un et l'autre sont Canadiens, Briand pourra justement qualifier leur départ de désertion. Ils ne reviendront jamais. Deux prêtres séculiers, anciens missionnaires d'Acadie, prennent la même route: Guillaume Cocquart et Philippe-Joseph Vizien; ce sont deux Français. Il part aussi des religieux: le récollet Gabriel Anheiser, âgé de 31 ans, se rembarque en même temps que les troupes; le jésuite Joseph-Pierre de Bonnecamp, âgé de 52 ans, en fait autant: l'un et l'autre sont Français.

Nous enregistrons d'autres départs en 1760. Le sulpicien François Picquet, âgé de 51 ans, et son confrère Charles Creitte, âgé de 28 ans; le récollet Ange Massot, âgé de 34 ans; les jésuites Pierre Audran, 39 ans, et Siméon Le Bansais, 41 ans, prennent le parti de rentrer en France où ils sont nés; et c'est probablement au cours de 1760 que le missionnaire Manach est renvoyé

\footnotetext{
1 Pour les références qui établissent les départs de 1759 et de 1760 , le lecteur voudra bien se reporter à notre Inventaire de l'Eglise canadienne à la fin de 1760 .
} 
d'Acadie en Angleterre. Enfin, la série des départs se clôt temporairement par le récollet Antoine Deperet, autre Français, âgé d'environ 30 ans: il signe son dernier acte à Chambly en janvier 1761 et nous sommes convaincu qu'il passe ensuite en France ${ }^{2}$.

De 1759 au début de 1761, l'Église canadienne avait donc vu partir 13 de ses membres, dont au moins six n'avaient pas encore 40 ans. A l'exception de nos deux chanoines, tous ceux qui partent sont Français de France; comme ils n'ont aucune attache au pays, ils n'hésitent pas à rentrer chez eux. Aucun de ces 13 émigrés n'a été contraint formellement de partir, même le sulpicien Picquet. Certes, Picquet, dont la tête avait naguère été mise à prix, avait ses raisons de partir, il ne tenait pas à subir le sort du malheureux Le Loutre, mais rien ne prouve qu'Amherst ait eu en 1760 l'intention de sévir contre lui. Le jésuite Audran avait peut-être intérêt à ne pas rester. Il avait déjà conduit les Abénaquis dans leurs terribles incursions ${ }^{3}$, mais le jésuite Roubaud, qui reste au Canada pendant le régime militaire, en avait fait autant.

Ces départs ne pouvaient qu'alarmer les autorités religieuses. L'abbé de L'Isle-Dieu, vicaire général des colonies de la NouvelleFrance, qui représentait en France l'Église canadienne, lance un appel pressant en 1762: "Mais surtout Messieurs (et je vous en conjure au nom du zele qui vous a animé jusque present) ne pensés pas a repassé en France dans les circonstances présentes et [...] abandonner un reste de troupeau qui a plus besoin de vous que jamais » ${ }^{4}$. A Forget du Verger, grand-vicaire pour le pays des Illinois, on adresse cet autre appel pressant: « Le pays que vous habités est cedé aux anglois et par cette consideration, Vous comprenés que votre présence et votre Ministère sont plus nécessaires que jamais au salut des âmes ${ }^{5}$. Mais les événe-

2 Sur Deperet, voir AOF-M, notes Jouve. Deperet n'a peut-être pas attendu l'été pour partir; il est fort possible qu'il soit parti par Boston ou par New-York.

3 L'Isle-Dieu à Mgr de Pontbriand, 25 mars 1755, dans RAPQ, 1936-37: 406.

4 L'Isle-Dieu au grand-vicaire Perrault, 17 mars 1762, ASQ, BM, 21728 (copie): 24.

s Villars à Forget du Verger, 14 mars 1763, ASQ, Missions, 35: 2 s. 
ments qui suivent la cession du Canada à l'Angleterre vont amener de nouveaux départs.

Le premier à partir est ce même Forget du Verger, prêtre séculier français et grand-vicaire des Illinois; le Séminaire de Québec lui avait confié la mission des Tamarois, à Cahokias, mission solidement établie et très bien pourvue. Forget du Verger, qui vivait pourtant dans le voisinage des Récollets et des Jésuites, se sentait abandonné et, quand il apprit la teneur du traité de Paris, il décida de tout vendre, esclaves, meubles et immeubles et de rentrer en France, malgré les instructions du Séminaire de Paris; Mgr Briand qualifiera ce départ de «honteuse retraite je dirois même criminelle ${ }^{6}$. Il n'y avait plus un seul prêtre séculier dans le pays des Illinois.

Mais ce même pays va subir un désastre religieux encore plus grand: quatre des six missionnaires jésuites vont être contraints de rentrer en France en 1764. Ce ne sont pas les Anglais qui les forcent à partir, mais ce sont les Français ! En effet, le Parlement de Paris en 1762 avait supprimé l'Ordre des Jésuites et confisqué ses biens; heureuses de se conformer à cette politique, les autorités françaises de la Louisiane émettent en juillet 1763 un mandat général d'expulsion contre les Jésuites. Elles ignorent encore que toute la rive occidentale du Mississipi a été cédée à l'Espagne; elles ignorent aussi, ou feignent de l'ignorer, que la rive orientale appartient désormais à l'Angleterre. On n'en procède pas moins contre les Jésuites, en vertu d'un ordre émanant de la métropole française, et cet ordre on l'applique non seulement contre les Jésuites qui venaient de la Louisiane, mais même contre ceux qui venaient du Canada. En cette année 1764, il part donc 4 jésuites: le P. Jean-Baptiste Lamorinie, âgé de 60 ans et missionnaire à Sainte-Geneviève; son compagnon, le $\mathrm{P}$. Jean-Baptiste de Salleneuve, âgé de 56 ans; le P. Philibert Watrin, vieillard de 67 ans, missionnaire aux Kaskaskias; le P. Julien Devernai, âgé de 45 ans, missionnaire à Vincennes. Il ne restera plus là que deux missionnaires: le $\mathrm{P}$. Aubert, qui sera

${ }^{6} \mathrm{Mgr}$ Briand au P. Meurin, s.j., lettre de 1767, citée dans Laval Laurent, Québec et l'Eglise aux Etats-Unis, 72, n. 9. Sur le départ de Forget du Verger, voir notre chapitre sur le Séminaire de Québec. 
d'ailleurs bientôt forcé de partir, et le P. Meurin qui se trouvera le seul missionnaire dans l'immense pays des Illinois et de la Haute-Louisiane ?.

Dans la vallée du Saint-Laurent, nous notons encore quelques départs, mais ils sont moins nombreux qu'à l'époque des capitulations. Notons ici le départ définitif d'un jésuite français de grands talents, Pierre-Antoine-Basile Roubaud, âgé de seulement 40 ans: réfugié dans la résidence de Murray depuis l'été 1763, Roubaud part de plein gré en juin 1764, officiellement dans le but d'aller se convertir en Europe dans quelque maison de son Ordre, mais en réalité pour accomplir au service de Murray une mission politique en Angleterre ${ }^{8}$.

Chez les prêtres séculiers, il se fait aussi des vides. Dominique Devoble, ancien missionnaire d'Acadie qui assume les fonctions curiales à Saint-Jean de l'île d'Orléans, signe son dernier acte le 21 juillet 1764 et rentre en France, son pays natal ${ }^{9}$. Mathurin-Joseph Jacrau, prêtre français d'environ 65 ans et procureur du Séminaire de Québec, a une santé tout à fait délabrée; il obtient la permission de passer en France pour s'y faire soigner; il met ordre à ses affaires et procède comme s'il n'allait jamais revenir: à la fin de septembre, il s'embarque pour la France. Son âge et sa santé rendent son retour tout à fait improbables, mais Jacrau reviendra ${ }^{10}$. Enfin, en ce même mois de septembre 1764, part le chanoine Jean-Olivier Briand: il a bien l'intention de revenir, car, choisi par le Chapitre pour remplir la vacance épiscopale, il se rend en Europe pour tenter de se faire accepter par l'Angleterre et se faire sacrer.

Un prêtre canadien vint bien près de partir: Charles Duchouquet, curé de Saint-Pierre-de-Montmagny. En 1760 et en 1761, par suite de l'occupation de son presbytère par des officiers anglais, Duchouquet avait subi toutes sortes de tribulations qui jetèrent un froid entre lui et le gouverneur Murray. Or, à l'occa-

${ }^{7}$ Sur le départ de ces jésuites, voir notre chapitre sur les Jésuites.

8 Sur Roubaud, voir notre même chapitre sur les Jésuites.

9 Registres de Saint-Jean (I.O.) ; notes manuscrites sur une édition de Tanguay (ASQ). Tanguay (Répertoire, 114) se trompe quand il le fait mourir à St-Jean en octobre 1765.

10 Sur Jacrau, voir notre chapitre sur le Séminaire de Québec. 
sion du peu d'enthousiasme que manifestaient les gens de la rive sud devant la campagne de recrutement des volontaires de 1764, Murray trouva lieu d'être mécontent de Duchouquet, sans qu'on sache exactement pourquoi. Sur les plaintes de Murray, Briand décide de retirer à Duchouquet une cure dont il jouissait depuis 14 ans. Duchouquet proteste de son innocence: "je Suis tres peiné des impostures contre moy [...] je nai jamais rien dit contre les ordres de $\mathrm{M}^{\mathrm{r}}$ le general au contraire, dans tous mes prones depuis un mois, je fesois mon possible pour exiter mes paroissiens a lexecution des dittes ordres [...] Si Mr le general se rapelloit ce que jai fais en 1760 et 1762 pour lexecution de Ses ordres, cela devroit Suffir pour me justifier, mais il faut perir innoncent Sans avoir la liberte de Se justifier ». Indigné de ces procédés et, de plus, menacé de perdre 2,000 livres dans la vente de ses meubles, il demande un exeat et une attestation «de vie et de mœurs » pour passer en France, lui qui était Canadien ${ }^{11}$. Il décidera finalement de rester au Canada, mais, toujours contraint de laisser sa cure de Saint-Pierre, nous le retrouvons à Lachenaie à partir de l'automne de $1764^{12}$. C'est sans doute l'attachement au pays natal qui avait eu le dessus.

Si nous comptons maintenant tous les départs que nous venons d'énumérer, nous arrivons au tableau suivant:

1759 le chanoine Charles-Ange Collet, Canadien le chanoine Gilles-Louis Cugnet, Canadien, 28 ans Guillaume Cocquart, Français Philippe-Joseph Vizien, Français le récollet Gabriel Anheiser, Français, 31 ans le jésuite Joseph-Pierre de Bonnecamp, Français, 52 ans

1760 Manach, Français

le sulpicien François Picquet, Français, 51 ans

le sulpicien Charles Creitte, Français, 28 ans

le récollet Ange Massot, Français, 34 ans

le jésuite Pierre Audran, Français, 39 ans

le jésuite Siméon Le Bansais, Français, 41 ans

11 Duchouquet à Briand, 10 avril 1764, AAQ, St-Pierre (Rivière-duSud), I, 17: 1s. Tanguay, op. cit., 110.

12 Il signe son dernier acte à St-Pierre le 15 septembre, et son premier à Lachenaie le 12 novembre. 
1761 le récollet Antoine Deperet, Français, 30 ans

1764 le chanoine Jean-Olivier Briand, grand-vicaire du Gouvernement de Québec, Français, 49 ans

Jacques-François Forget du Verger, grandvicaire des Illinois, Français

Dominique Devoble, Français

Mathurin-Joseph Jacrau, Français, 65 ans

le jésuite Jean-Baptiste Lamorinie, Français, 60 ans

le jésuite Jean-Baptiste de Salleneuve, Français, 56 ans

le jésuite Philibert Watrin, Français, 67 ans

le jésuite Julien Devernai, Français, 45 ans

le jésuite Pierre-Antoine-Basile Roubaud, Français, 40 ans.

De 1759 à la fin de 1764, nous comptons donc 22 prêtres qui passent en France: 3 chanoines, 6 prêtres séculiers, 2 sulpiciens, 3 récollets et 8 jésuites. Remarquons toutefois dans cette liste le chanoine Briand qui se rend en Europe pour tenter de s'y faire sacrer et Jacrau qui va se faire soigner: l'un et l'autre ont l'intention de revenir. Il reste donc 19 départs définitifs. Sauf les deux chanoines Collet et Cugnet, tous ceux qui partent pour de bon sont des Français. Les jésuites sont les plus nombreux, et c'est un grand malheur puisque la Compagnie de Jésus n'a plus l'autorisation de se recruter.

Mais ces 19 départs définitifs ne sont pas tous des départs volontaires: le séculier Manach, missionnaire d'Acadie, part bien malgré lui; la situation difficile dans laquelle se trouve le sulpicien Picquet, lui vaut une excuse légitime; les quatre jésuites des Illinois sont contraints par les autorités françaises de rentrer dans leur pays. Sur les 19 prêtres qui rentrent en France pour ne plus revenir, il y en a donc 13 qui paraissent partir de leur plein gré, sans y être contraints par une force majeure. C'est ce chiffre 13 qu'il faut retenir lorsque, en examinant l'inventaire de l'Église au début de 1759 , on veut savoir s'il y a eu exode du clergé.

Maintenant que nous nous sommes arrêtés à ce chiffre 13, pouvons-nous vraiment parler d'un exode du clergé? Si nous 
comparons ce chiffre au total des 196 prêtres que nous trouvions au pays au début de 1759 , nous constatons que ces 13 prêtres qui, de plein gré, partent définitivement, ne constituent qu'une faible proportion de $6.6 \%$ : autant dire qu'il n'y a pas eu exode du clergé et que les départs volontaires et définitifs ne sont que de rares exceptions.

Mais, pour apprécier exactement les pertes que subit l'Église canadienne pendant cette période, il reste quand même qu'on doit compter aussi bien les départs forcés que les départs volontaires. Nous avons énuméré 22 prêtres qui partent; qu'ils aient l'intention plus ou moins précise de revenir, qu'ils comptent demeurer indéfiniment en France, peu importe ici: à cette époque, celui qui part pour l'Europe, n'est pas sûr de pouvoir revenir. Ce total de 22 départs constitue une proportion déjà un peu élevée de $11.2 \%$.

\section{Les nombreux vides causés par les décès}

En elle-même, assurément, cette proportion de $11.2 \%$ ne serait pas inquiétante si elle représentait à elle seule toutes les pertes subies par l'Église canadienne, mais il faut la mettre en regard d'une autre cause qui crée impitoyablement des vides. Du début de 1759 à la fin de 1764, il meurt bien des prêtres et pas des plus vieux. Le tableau qui suit, est d'une longueur effarante ${ }^{13}$ :

1759 Sébastien Duguay, Français, décédé en mars,

à l'âge de 40 ans

Louis Chardon, Français, décédé le 13 mars

Joseph Gagnon, Français, décédé en juillet

René Portneuf, Canadien, mort à la guerre le 23 août, à l'âge de 52 ans

le sulpicien Louis Normant, Français, décédé

le 18 juin, à l'âge de 78 ans

le récollet Salvien Boucher, Canadien, décédé à l'âge de 58 ans

le récollet Maurice Imbault, Français, décédé

à l'âge de 73 ans

13 Pour les années 1759 et 1760 , nous n'indiquons aucune référence: le lecteur voudra bien se reporter à notre inventaire de l'Eglise canadienne à la fin de 1760 . 
le jésuite Claude-Joseph Virot, Français, mort à la guerre le 24 juillet, à l'âge de 37 ans

le jésuite Nicolas Degonnor, Français, décédé le 16 décembre, à l'âge de 68 ans.

1760 Gaspard Dunière, Canadien, décédé le 2 février, à l'âge de 41 ans

Jean-Baptiste La Coudray, Canadien, décédé le 5 mai, à l'âge de 62 ans

Jean-Charles Chevalier, Français, décédé le 18 mai, à l'âge de 66 ans

l'évêque Henri-Marie Dubreil de Pontbriand, Français, décédé le 8 juin, à l'âge de 51 ans

Pierre Chaufour, Canadien, inhumé le 20 juillet, à l'âge de 36 ans

le chanoine Michel Poulin, Canadien, décédé le 10 octobre, à l'âge de 72 ans

Charles Dubergé, Français, décédé au cours de l'automne, à l'âge de 31 ans

François Roüillard, Canadien, décédé le 15 décembre, à l'âge de 59 ans

le sulpicien Joseph Hourdé, Français, décédé le 10 mai, à l'âge d'environ 72 ans

le récollet Justinien Constantin, Canadien, décédé le 22 mars, à l'âge de 44 ans

le récollet Etienne Cotton, Canadien, décédé à l'âge de 29 ans

le jésuite Jacques-François Le Sueur, Français, décédé en avril, à l'âge de 75 ans

1761 Charles-Louis-Marie Baudoüin, Canadien, décédé le 4 février, à l'âge de 44 ans ${ }^{14}$

Pierre Leclair, Français, décédé le 26 novembre, à l'âge de 74 ans ${ }^{15}$

le sulpicien Antoine Déat, Français, décédé en mars, à l'âge de 65 ans ${ }^{16}$

le jésuite Jean-Baptiste Deneuville, Français, décédé le 15 janvier, à l'âge de 39 ans ${ }^{17}$

le jésuite Etienne Lauverjat, Français, décédé le 16 novembre, à l'âge de 82 ans ${ }^{18}$

14 Tanguay, Répertoire, 118.

15 Ibid., 92 ; Sirois,Cap-St-Ignace, 34; Allaire, Dictionnaire, III : 41.

16 Tanguay, op. cit., 98 .

17 RJ, LXXI: 177.

18 Ibid., 163. 
1762 Pierre Petit, Canadien, inhumé le 10 mars, à l'âge de 34 ans ${ }^{19}$

Pierre Maillard, grand-vicaire de l'Acadie, Français, décédé probablement le 12 août 20

Louis-Nicolas Godefroy Normanville, Canadien, décédé le 3 octobre à l'âge de 38 ans ${ }^{21}$

Louis Lepage de $\mathrm{S}^{\text {te }}$ Claire, Canadien, décédé en décembre, à l'âge de 71 ans ${ }^{22}$

le jésuite Alexis-Xavier de Guyenne, Français, décédé à l'âge de 66 ans ${ }^{23}$

1763 Jean-Baptiste Dugast, Canadien, décédé le 9 mai, à l'âge de 79 ans ${ }^{24}$

Joseph Ysambart, Français, décédé le 14 décembre, à l'âge de 70 ans ${ }^{25}$

le sulpicien Mathieu Falcoz, Français, inhumé le 14 avril, à l'âge de 61 ans et demi ${ }^{26}$

1764 François Martel, Canadien, décédé le 2 février, à l'âge de 58 ans ${ }^{27}$

Dominique-Joseph Lacombe, Canadien, décédé le 27 juin, à l'âge de 63 ans ${ }^{28}$

le sulpicien Hamon Guen, Français, décédé en avril, à l'âge de 77 ans ${ }^{29}$

le récollet Hippolyte Collet, Français, décédé vers la fin de l'année, à l'âge de 72 ans ${ }^{30}$

le jésuite Simon-Pierre Gounon, Français, noyé le 3 mai, à l'âge de 45 ans ${ }^{31}$.

19 Registres de St-Michel-de-Bellechasse; Tanguay, op. cit., 127.

20 Burns, The Abbé Maillard and Halifax, dans Soc. can. hist. Eglise, 1936-37: 13-22.

21 Registres de Notre-Dame-de-Montréal, 4 octobre 1762.

22 Mort le 3 décembre 1762, selon Gareau, Terrebonne, 29. Le 25 janvier 1763, Montgolfier écrit que Lepage est mort depuis plus d'un mois (Montgolfier à Briand, AAQ, Cartable des grands vicaires, 25).

23 RJ, LXXI: 169.

24 Registres de St-François-du-Lac, 11 mai 1763.

25 Mère d'Youville à Montgolfier, 2 janvier 1764, dans Ferland-Angers,

Mère d'Youville, app.: 219.

26 Registres de Notre-Dame-de-Montréal, 14 avril 1763.

27 Registres de St-Laurent de l'île d'Orléans, 4 février 1764. Tanguay se trompe en le faisant mourir en 1763 (op. cit., 108).

28 Tanguay, op. cit., 102.

29 Gauthier, Sulpitiana, 213.

30 Voir ce que nous en disons au chapitre des Récollets.

31 Haldimand à Gage, 27 juin 1764, BM, 21662: 151v.; RJ, LXXI : 177. 
Chaque année, surtout en 1759 et en 1760, il disparaît ainsi des membres du clergé: 1759,$9 ; 1760,12$ (y compris l'évêque); 1761,$5 ; 1762,5 ; 1763,3 ; 1764,5$. Le nombre des prêtres décédés s'élève à 39 en l'espace de cinq ans ! De ce nombre, 23 sont des Français de France, c'est-à-dire les 59\%: à cause de la cession définitive du Canada, on ne peut plus compter sur le clergé de France pour les remplacer; quant aux Canadiens, il n'y a pas encore d'évêque pour leur donner des successeurs et on ne sait pas encore si on va parvenir à obtenir un évêque.

Le point le plus alarmant de ce tableau, c'est le grand nombre de jeunes prêtres qui disparaissent: 11 n'ont pas encore 45 ans, et, de ce nombre, il y en a même un, le récollet Cotton, qui n'a pas 30 ans. En cette difficile époque de transition, l'Église canadienne devait surtout compter sur la jeunesse de ses membres pour durer le plus longtemps, au cas où la question épiscopale tarderait à se régler.

Les décès se répartissent comme suit:

22 chez les séculiers
7 chez les Jésuites
5 chez les Récollets
5 chez les Sulpiciens.

Toute proportion gardée, ce sont les Jésuites qui ont le plus perdu par les décès; le pourcentage des pertes est le suivant:

$22.6 \%$ chez les Jésuites

$21.8 \%$ chez les séculiers

$16.7 \%$ chez les Récollets

$14.7 \%$ chez les Sulpiciens.

Pour cette période de cinq ans, le nombre seul des décès est alarmant; or il y avait eu aussi des départs pour l'Europe et ces départs se chiffraient au total de 22. Si nous ajoutons ces départs aux décès, nous constatons que l'Église canadienne, en cinq ans, se voit privée de 61 de ses membres: la proportion des pertes totales est de $31.1 \%$ ! Si réunissant les départs et les décès, nous considérons les pertes totales de chacun des groupes, nous trouvons les proportions suivantes:

$48.4 \%$ chez les Jésuites

$30.7 \%$ chez les séculiers 


\section{$26.7 \%$ chez les Récollets $20.6 \%$ chez les Sulpiciens.}

Les Jésuites perdent donc, en cinq ans, près de la moitié de leurs effectifs: c'est assurément un désastre et, à cause des persécutions dont on accable les Jésuites dans le monde, ce désastre sera irréparable. Chez les séculiers, la proportion n'atteint pas tout à fait le tiers, mais, vu les circonstances, cette proportion est aussi désastreuse; elle ne l'est pas moins chez les Récollets, puisqu'après avoir perdu $26.7 \%$ de leurs effectifs, ils reçoivent, comme les Jésuites, la défense de se recruter.

\section{Le tiers des effectifs demeure à peu près perdu}

L'Église canadienne a donc perdu, en cinq ans, le tiers de ses effectifs. Cette perte effarante est-elle corrigée au moins de quelque façon? L'Église du Canada était en droit de compter au moins sur ses prêtres canadiens qui, pour diverses raisons, n'étaient plus au pays; elle avait droit d'espérer qu'ils reviennent.

Le chanoine Joseph-Marie Lacorne, prêtre canadien, était parti en 1750 pour s'occuper en France des affaires du Chapitre: le roi l'avait nommé doyen en 1755 . En 1763, grâce à ses démarches très actives à Londres, on obtient que l'Angleterre ferme les yeux sur l'épiscopat: c'est lui qui est à l'origine du règlement délicat de la question épiscopale; il faut lui laisser son mérite. Cependant, le chanoine Lacorne est bien décidé de ne pas revenir. Le 2 mai 1763, il écrivait à lord Orwell: " Ayant renoncé depuis longtemps a retourner en Canada meme dans le cas ou il Seroit resté a la france ${ }^{32}$. Il ne changea rien à cette décision. Ses deux confrères, Charles-Ange Collet et Gilles-Louis Cugnet, l'un et l'autre Canadiens, décident eux aussi de rester en France pour de bon, méritant ainsi parfaitement le mot désertion que leur appliquera Mgr Briand ${ }^{33}$. Si le chanoine Lacorne avait déjà 50 ans, Cugnet n'en comptait que 28. Le vicaire général l'Isle-

32 Lacorne à Orwell, 2 mai 1763, AAQ, D. Q., I, $59: 4$.

$33 \mathrm{Mgr}$ Briand au cardinal Castelli, 3 mars 1766, AAQ, Evêques de Québec, I, 114. 
Dieu, pour sa part, regrettera que ces trois Canadiens trouvent * plus commode, plus agréable et moins penible de vivre en France », à une époque où l'Église canadienne ne pouvait plus compter sur les prêtres français ${ }^{34}$.

Un autre prêtre canadien opte définitivement pour la France, Pierre-François de Thiersant; il n'avait que 40 ans. Passé en France avant la conquête, il revient au Canada mettre ordre à ses affaires. Nous notons sa présence à Saint-François-duLac le 7 août 1763, lorsqu'il signe deux actes dans les registres paroissiaux; il est encore au pays le 1er mai 1764 : ce jour-là, il comparaît devant la Chambre des Milices de Montréal; le 4 mai, il s'entend avec le Séminaire de Montréal sur les limites d'une concession; enfin, le 11 octobre, il vend ses seigneuries à un Anglais et rentre en France ${ }^{35}$. Ce prêtre, fils d'une riche famille bourgeoise, trouve plus commode, lui aussi, de continuer sa vie en France. C'est un autre déserteur.

Il y avait encore en France au moins quatre prêtres canadiens. Jean-Louis Lacorne, frère jumeau du chanoine Lacorne, avait été récollet sous le nom de $P$. Maurice: rentré en France, il se sécularise et, comme son frère, $\mathrm{y}$ demeure pour de bon ${ }^{36}$; en 1764, il n'a que 50 ans. Le récollet canadien Michel Levasseur est, lui aussi, passé en France et ne revient pas, mais nous ne sommes pas tout à fait sûr qu'il soit encore vivant en 1764 ; s'il l'est, il a 65 ans ${ }^{37}$ : ce serait presque un vieillard, mais le Français Jacrau aura le courage de passer au Canada en 1766, à l'âge de 68 ans ... Il y a en France un troisième prêtre canadien qui ne revient pas, c'est le jésuite François-Xavier Duplessis, célèbre prédicateur du dix-huitième siècle, mais son retour ne serait peut-être qu'une nouvelle charge pour la communauté canadienne,

34 L'Isle-Dieu au Préfet de la Propagande, 19 juin 1766, AAQ, Archives du Vatican, Eglise du Canada, VII: 231s.

$35 \mathrm{~A}$ St-François-du-Lac, il signe de thiersant de Bourgmarie; à SteAnne-de-Beaupré, il avait signé $p$. $f$. de thiersant (voir les registres). Sur son procès de 1764, voir APQ, Registre des Sentences, 1764, f. 1. Il possédait les seigneuries Bonsecours, Bourgmarie et St-Charles dans le Gouvernement de Montréal. Sur lui, voir $\mathrm{H}$. Lapalice, Histoire de la seigneurie Massue, 81-85.

${ }_{36}$ AOFM, notes Jouve.

37 Loc. cit. 
puisque ce jésuite atteint ses 70 ans ${ }^{38}$. Un quatrième prêtre canadien tarde à rentrer en son pays, mais il s'agit ici d'un triste sire: François-Louis Chartier de Lotbinière, récollet, était parti du Canada en 1753, interdit et suspens de tous les ordres; devenu cordelier, puis, de nouveau, récollet, il avait vécu en apostat pendant deux ans; enfin, il était devenu frère servant de l'Ordre de Malte; un trop fort penchant pour l'eau-de-vie semble avoir causé tous ses malheurs. Nous le verrons réapparaître au pays en $1769{ }^{39}$. Enfin, l'Église du Canada serait encore en droit de compter sur le seul confrère canadien du jésuite Duplessis: Michel Baudoüin, grand-vicaire de la Louisiane, mais le P. Baudoüin, âgé de 72 ans, est un vieillard réduit à l'incapacité presque totale ${ }^{40}$.

En France, il reste encore quelques recrues canadiennes sur lesquelles on espère pouvoir compter un jour, ce ne sont encore que de jeunes séminaristes: Jean Bro, âgé de 21 ans, et Mathurin Bourg, âgé de 20 ans, achèvent leur Philosophie au séminaire du diocèse de Saint-Malo; Jean-Pierre Bourg, 22 ans, et Isaac Hébert, 17 ans, terminent leurs humanités ${ }^{41}$. Les deux premiers seulement, Jean Bro et Mathurin Bourg, répondront à l'espoir de l'Église canadienne.

Dans la période à laquelle nous limitons notre étude, 17591764 , il n'y a que deux prêtres canadiens qui rentrent de France pour se donner définitivement à l'Église canadienne: JosephNicolas Martel et Pierre Mennard. Martel, entré chez les Jésuites, était passé en France pour y subir sa formation; en 1763, il

38 J.-Edmond Roy, Notice biographique, dans Lettres du P. F.-X. Duplessis, V-LXXXV. L'auteur reproduit (p. XIV, n. 2) l'acte de baptême de ce jésuite. Voir fac-similé de sa signature, p. LXXVII.

$39 \mathrm{Mgr}$ Briand à Chartier de Lotbinière, frère de celui dont nous parlons, 13 juillet 1769, AAQ, Copies de lettres, IV : 297; Mgr Briand, 2 octobre 1770, AAQ, Registre C, 251; Mgr Briand à L'Isle-Dieu, 7 juin 1774, AAQ, Evêques de Québec, I: 172. Sur lui, voir Laval Laurent, Québec et l'Eglise aux Etats-Unis, 54, n. 133. A St-Laurent, en 1770, il signe parfois chartier de Lotbiniere cure miss. pretr. de Lordre de malte; habituellement, il signe Lotbiniere.

40 RJ, LXXI: 169; il signe M. Baudoüin jesuite (RAPQ, 1936-37: 393).

41 L'Isle-Dieu au Préfet de la Propagande, 21 juin 1766, AAQ, Archives du Vatican, Eglise du Canada, VII : 249. Ils étaient Acadiens. Nous corrigeons les âges indiqués par l'Isle-Dieu en recourant à des pièces publiées dans RAC, 1905, II, app. A, 3: 336-339. 
quitte la Compagnie de Jésus et arrive au pays, paraît-il, le 6 juin 1764 ; il aurait pris charge de Saint-Laurent de l'île d'Orléans, dont le curé, son frère, venait de mourir ; à partir de décembre, il signe à Saint-Jean de l'île d'Orléans avec le titre de curé. Mais cette nouvelle recrue a déjà 43 ans ${ }^{42}$.

Le même jour que Joseph-Nicolas Martel, du moins selon l'affirmation de Tanguay ${ }^{43}$, Pierre Mennard débarque à Québec. L'historien Gosselin en a fait un Breton ${ }^{44}$, mais Mennard était bien Canadien. Né en 1739 de Jean-Louis Mennard et de MartheMarguerite French, cet étudiant montréalais étudie au Collège des Jésuites où, vers 1758 , il soutient ses thèses de philosophie. Au cours de 1759, il porte les armes; puis, on l'envoie en France pour y poursuivre ses études, en même temps qu'un autre jeune Canadien que nous n'avons pu identifier; en décembre 1760, il débarque à La Rochelle "sans hardes, sans Linge, et dépourvu géneralement de tout ». Les Messieurs de Paris l'envoient étudier dans le diocèse de Bayonne; au nom du Séminaire de Québec, ils l'habillent, lui paient sa pension et versent même 110 livres pour les frais d'une absolution a gestatione armorum qu'il a fallu obtenir de Rome. En 1763, il passe à Paris, au Séminaire de Saint-Nicolas. Le 7 avril 1764, il est ordonné prêtre; l'abbé de l'Isle-Dieu écrit à Mère d'Youville: «il fut ordonné Samedy dernier veille du Dimanche de la passion, Et il paroit avoir un très grand empressement et beaucoup d'envie de se rendre dans son pays natal, ou je crois qu'on sera content de lui, c'est un fort joli sujet. » Comme l'annonçait l'Isle-Dieu, Mennard part probablement le 13 avril; il se met en route pour Londres d'où il s'embarque pour Québec. Que fait-il dans les premiers mois qui suivent son retour? Il se peut qu'on l'ait gardé au Séminaire de Québec; l'Isle-Dieu avait d'ailleurs écrit: «M. Menard m'a paru porter à passer au moins un an avec vous au seminaire et je le Désire afin qu'il se forme aux fonctions du $\mathrm{s}^{\mathrm{t}}$ ministere ». Com-

42 Tanguay, Répertoire, 136; RJ, LXXI: 181. Comme il manque une partie des registres à St-Laurent, nous n'avons pu constater si JosephNicolas Martel y signe des actes. Le 7 décembre 1764, il signe son premier acte à St-Jean; sa dernière signature en cet endroit est du 6 mai 1767. Il lui arrive de signer Jos. Nic. martel ptre.

43 Tanguay, op. cit., 122.

44 Gosselin, L'Eglise du Canada après la conquête, II : 90. 
me Mennard arrive à Québec aussi dénué qu'à La Rochelle, le Séminaire lui prête 308 livres en septembre. Mennard entre bientôt dans le ministère actif : à partir du 27 octobre 1764, il signe régulièrement dans les registres de Notre-Dame-de-Québec, avec le titre de vicaire, et il porte ce titre jusqu'au 21 septembre 1766; après quoi, on l'envoie à Saint-Jean de l'île d'Orléans ${ }^{45}$ Ce cas Mennard est intéressant, non seulement parce qu'il a donné lieu à des recherches assez longues pour corriger Tanguay et pour suivre les allées et venues de cet étudiant en France, mais aussi parce que nous avons là le cas presque unique d'un jeune Canadien qu'on envoie se faire ordonner outre-mer parce qu'il n'y a plus d'évêque. L'Église canadienne ne pouvait guère répéter ce procédé, puisque pour le seul Mennard il en avait coûté plus de 2,000 livres.

Pour remplacer les 22 prêtres passés en Europe et les 39 prêtres décédés, on ne trouve donc que deux sujets: Martel et Mennard.

Il y a cependant au pays même quelques ecclésiastiques qui se préparent à la prêtrise. Au Séminaire de Québec, en cette fin d'année 1764, nous trouvons Jean-Baptiste Corbin, FrançoisXavier Dézéry, Pierre Gibault, Jean-François Hubert (qui fait les fonctions d'économe), Pierre-René Hubert et Jean-Marie Verreau. Il en est resté au Séminaire de Montréal: Pierre Denault, qui continue de servir de secrétaire au grand-vicaire de Montréal; Jean-Baptiste Pouget, Pierre-Mathieu Gamelin-Maugras et peut-être. Charles-François Bailly de Messein ${ }^{46}$. Mais qu'est-ce qu'une dizaine d'ecclésiastiques pour compenser une soixantaine de pertes ? Et d'ailleurs, rien de moins certain que l'avenir de ces ecclésiastiques, puisqu'on ne sait pas encore si le pays obtiendra jamais un évêque.

45 Sur le cas Mennard, voir notre article Note supplémentaire sur messire Pierre Mennard, Canadien, dans RHAF, VII, 2 (septembre 1953): 290-292; comme aussi les articles de Jean-Jacques Lefebvre, ibid., VI, 4 (mars 1953): 560-563; VII, 2 (septembre 1953): 290-292; et l'article d'Albertine Ferland-Angers, ibid., 287-290. Dans une lettre personnelle, madame Ferland-Angers nous a communiqué des détails qui nous permettent ici de préciser ou mếme de rectifier certains petits points de notre article. La solution du cas Mennard est le résultat de la collaboration.

46 Sur ces ecclésiastiques, voir, dans la deuxième partie de cet ouvrage, notre chapitre sur le Séminaire de Québec. 
Compter sur le recrutement de prêtres français? on ne peut absolument plus y songer, du moins pour quelques années; les chanoines l'ont écrit à l'automne de 1764: «Le General Muray a Déclaré a Mr Mongolfier quil Ne Souffriroit jamais qu'il vint En Canada aucun Prestre francois tant quil Seroit gouverneur ${ }^{47}$.

\section{Dénombrement de l'Église canadienne à la fin de 1764}

22 prêtres passés en Europe, 39 décédés : voilà pour les pertes; 2 prêtres canadiens qui rentrent, voilà tout ce qu'on reçoit. Le déficit est effarant. Mais voyons les membres qui composent cette Église canadienne, en cette fin d'année 1764. Nous procéderons comme nous l'avons fait pour 1760 , car il importe de voir en même temps comment le clergé est distribué à travers le pays.

\section{Acadie}

Louisbourg et île Saint-Jean, pas un seul missionnaire.

Acadie péninsulaire, pas un seul missionnaire depuis la mort de Pierre Maillard.

Rivière Saint-Jean, pas un seul missionnaire depuis le départ du jésuite Germain ${ }^{48}$.

Miramichy, le récollet Bonaventure Carpentier, Canadien, âgé de 48 ans $^{49}$ : il est le seul prêtre catholique à exercer le ministère dans cette partie du pays.

Ristigouche, pas un seul missionnaire depuis le départ du récollet Rouillard ${ }^{50}$.

\footnotetext{
47 Le chapitre au doyen Lacorne, lettre de l'automne 1764, AAQ, Gouvernement, V, 19: 2 .

${ }^{48}$ Le P. Germain a dû quitter sa mission de la rivière St-Jean en 1762 ; nous le retrouvons au Cap-de-la-Madeleine où il signe son premier acte le 5 janvier 1763 .

49 Là où nous ne donnons pas de références pour les détails biographiques, pour la localisation des prêtres et pour l'inventaire des églises ou chapelles, on voudra bien se reporter aux références que nous donnons dans notre chapitre, Inventaire de l'Eglise canadienne à la fin de 1760. Quant aux jésuites, aux récollets et aux sulpiciens, on se reportera aux chapitres que nous avons écrits sur eux.

50 Le cahier des registres s'interrompt après le 5 mai 1761 et ne reprend qu'en 1771. Rouillard se fixe apparemment à Rimouski.
} 


\section{Gouvernement de Québec ${ }^{\text {Б1 }}$}

Sept-Iles, point de prêtre résidant.

Les Islets-Jérémie, point de prêtre résidant.

Chicoutimi, point de prêtre résidant.

Tadoussac, le jésuite Claude Coquart, Français, âgé de 58 ans; il dessert l'immense région qui s'étend des Islets-Jérémie aux Eboulements.

La Malbaie, point de prêtre résidant.

Les Eboulements, point de prêtre résidant ${ }^{52}$.

Ile-aux-Coudres, point de prêtre résidant.

Baie-Saint-Paul, Louis Chaumont, âgé d'environ 64 ans; il dessert en même temps l'lle-auxCoudres et la Petite-Rivière-Saint-Francois.

La Petite-Rivière-Saint-François, point de prêtre résidant.

Saint-Joachim, Bernard-Sylvestre Dosque, Français, âgé d'environ 44 ans ${ }^{53}$.

Sainte-Anne-de-Beaupré, Louis-Laurent Parent, Canadien, âgé de 41 ans.

Château-Richer, François Duburon, Canadien, âgé de 37 ans.

L'Ange-Gardien, Michel Marcou, Canadien, âgé de 30 ans.

Saint-Pierre (I.O.), Louis-Philippe Desgly, Canadien, âgé de 54 ans.

Sainte-Famille (I.O.), Gilles Eudo, Français, âgé de 40 ans.

Saint-François (I.O.), François Le Guerne, Français, âgé de 40 ans.

Saint-Jean (I.O.), point de prêtre résidant ${ }^{54}$.

51 Depuis septembre 1764 , il n'y a plus, à proprement parler, de Gouvernement de Québec, Gouvernement des Trois-Rivières ni Gouvernement de Montréal: le pays a été divisé en deux districts; mais nous conservons quand même la division en Gouvernements parce qu'elle nous a servi de base lors de notre inventaire de 1760 .

52 C'est le jésuite de la Brosse qui signe les registres à la fin de 1764, mais il n'était là que de passage: sa résidence habituelle est à Mascouche, et c'est pourquoi nous l'énumérons à Mascouche.

53 Il signe son dernier acte à Beaumont le 19 octobre 1761 et, à partir du 31 octobre, il signe habituellement les registres de St-Joachim.

54 Le Français Devoble signe son dernier acte le 21 juillet 1764 et se.prépare à rentrer pour de bon en France: la paroisse reste sans prêtre et doit compter sur le curé de la paroisse voisine. De Saint-Laurent, Joseph- 
Saint-Laurent (I.O.), Joseph-Nicolas Martel, Canadien, âgé de 43 ans ${ }^{85}$.

Beauport, Pierre-Simon Renauld, Canadien, âgé de 33 ans.

Jeune-Lorette, le jésuite Etienne-Thomas-deVilleneuve Girault, Français, âgé de 46 ans.

Charlesbourg, Jean-Baptiste-Laurent Morisseaux, Canadien, âgé de 46 ans.

Québec

Notre-Dame-de-Québec, depuis la Noël de 1764, les offices de la paroisse ont lieu dans la chapelle du Séminaire; le ministère paroissial est assuré par deux prêtres:

Jean-Félix Récher, Français, âgé de 40 ans, curé de la paroisse et chanoine honoraire;

Pierre Mennard, Canadien, âgé de 25 ans.

Séminaire, Urbain Boiret, Français, Supérieur ;

Colomban-Sébastien Pressart, Français, âgé de 41 ans;

Henri-François Gravé, Français, âgé de 34 ans.

Collège des Jésuites, le jésuite Augustin-Louis

De Glapion, Français, âgé de 45 ans, supérieur général;

le jésuite René-Pierre-Daniel Richer, Français, âgé de 82 ans;

le jésuite Marin-Louis Lefranc, Français, âgé de 48 ans.

Couvent des Récollets, le récollet Emmanuel

Crespel, Français, âgé de 61 ans, Commissaire provincial;

le récollet Didace Cliche, Canadien, âgé de 54 ans;

Nicolas Martel vient y signer les registres du 7 décembre 1764 au 6 mai 1767: il s'intitule tantôt prêtre desservant de St-Jean, tantôt curé de St-Laurent. A l'examen des registres, on se rend vite compte que Martel n'y vient que de temps à autre.

55 François Martel signe son dernier acte à St-Laurent le 14 janvier 1764, puis il meurt le 2 février. La paroisse demeure sans prêtre pendant quelques mois: c'est Desgly, curé de St-Pierre, qui vient signer les actes. Joseph-Nicolas Martel, frère du feu curé, arrive d'Europe et vient immédiatement occuper la cure de St-Laurent et dessert en même temps la paroisse de St-Jean. Pour notes biographiques de Martel, voir Tanguay, Répertoire, 136; RJ, LXXI : 181. 
le récollet Jean-Baptiste Lajus, Canadien, âgé de 60 ans ;

le récollet Daniel Normandin, Canadien, âgé de $\mathbf{5 8}$ ans;

le récollet Augustin Quintal, Canadien, âgé de 81 ans;

le récollet Alexis DuBuron, Canadien, âgé de 45 ans.

Couvent des Ursulines, le chanoine Joseph Resche, Canadien, âgé de 69 ans.

Hôpital Général, le chanoine Joseph-François Perrault, Canadien, âgé de 45 ans; grandvicaire du Gouvernement de Québec;

le chanoine Charles-Régis Rigauville, Canadien, âgé de 42 ans; il fait les fonctions de chapelain de l'Hôpital et de curé de Notre-Dame-des-Anges.

Sainte-Foy, François Borel, Français, âgé de 37 ans.

L'Ancienne-Lorette, François-Ignace Levasseur, Canadien, âgé de 56 ans.

Saint-Augustin, François-Xavier-Nicolas-Marie Brassard, Canadien, âgé de 43 ans.

Neuville, Louis-Eustache Chartier de Lotbinière, Canadien, âgé de 49 ans.

Les Ecureuils, Louis-Michel Beriau, Canadien, âgé de 36 ans.

Cap-Santé, Joseph Fillion, Canadien, âgé de 38 ans.

Deschambault, Jean Ménage, Canadien, âgé de 80 ans.

Les Grondines, point de prêtre résidant ${ }^{56}$.

Deschaillons, Jacques Hingan, Français, âgé de 35 ans.

Lotbinière, Jean-Baptiste Gatien, Canadien, âgé de 45 ans ${ }^{57}$.

Ste-Croix-de-Lotbinière, le récollet François Carpentier, Canadien, âgé de 54 ans.

56 Jacques Hingan y signe son dernier acte le 30 septembre 1761 ; le récollet Louis Demers vient y signer des actes à partir du 6 janvier 1762; le 11 juillet 1764, c'est le curé de Ste-Anne-de-la-Pérade, Guay, qui assure la desserte.

57 Gatien y signe son premier acte le 11 novembre 1764 . 
St-Antoine-de-Tilly, Jean-Baptiste Noël, Canadien, âgé d'environ 54 ans.

St-Nicolas, Ignace Desroches, Canadien, âgé de 36 ans.

St-Joseph-de-Beauce, le récollet Théodore Loiseau, Français, âgé de 38 ans; il dessert en même temps Ste-Marie et St-François.

Ste-Marie-de-Beauce, point de prêtre résidant.

St-François-de-Beauce, point de prêtre résidant ${ }^{58}$.

St-Henri, point de prêtre résidant ${ }^{59}$.

La Pointe-Lévy, Charles Youville-Dufrost, Canadien, âgé de 35 ans ${ }^{60}$.

Beaumont, Pierre Parent, Canadien, âgé de 31 ans ${ }^{61}$.

St-Charles-de-Bellechasse, Louis Sarault, Canadien, âgé de 38 ans.

St-Michel, le récollet Emmanuel Veyssière, Français, âgé de 30 ans.

St-Vallier, Thomas Blondeau, Canadien, âgé de 55 ans ${ }^{62}$.

Berthier-en-bas, Antoine Lagroix, Canadien, âgé de 44 ans ${ }^{63}$.

St-François-de-Montmagny, Pierre-Laurent Bédard, Canadien, âgé de 35 ans.

St-Pierre-de-Montmagny, Jean-François Curot, Canadien, âgé de 40 ans ${ }^{64}$.

St-Thomas-de-Montmagny, Jean-Baptiste Maisonbasse, Français, âgé de 43 ans.

58 Il $\mathrm{y}$ avait une chapelle à Ste-Marie, mais les actes étaient insérés dans le cahier des registres de St-Joseph. St-François avait aussi sa chapelle, mais elle était toute récente: on y fait le premier baptême le 11 novembre 1763, mais les actes sont encore insérés dans le cahier des registres de St-Joseph.

59 La paroisse est desservie par le curé de la Pointe-Lévy.

$60 \mathrm{De}$ retour de son exil de Ste-Rose, il signe son premier acte le 15 novembre 1761 .

61 Il signe les actes de Beaumont du 24 février 1762 au 15 janvier 1765.

62 Parti de Berthier-en-bas, il signe son premier acte à St-Vallier le 24 janvier 1762.

63 Après avoir signé son dernier acte à Ste-Geneviève-de-Batiscan le 16 octobre 1761, il passe à Lotbinière tout de suite après; il signe son dernier acte à cet endroit le 29 octobre 1764 pour se rendre à Berthier-en-bas dont il signe les actes du 13 novembre 1764 au 9 décembre 1765 .

64 Duchouquet, renvoyé de St-Pierre par l'ordre de Murray, signe son dernier acte le 15 septembre 1764 ; puis, la paroisse est desservie par le curé de St-François; à partir du 3 octobre, c'est Curot qui signe les actes. 
Cap-St-Ignace, Charles Gareault, Canadien, âgé de 40 ans ${ }^{65}$.

L'Islet, Romain Dolbec, Canadien, âgé de 47 ans.

St-Jean-Port-Joly, point de prêtre résidant ${ }^{66}$.

St-Roch-des-Aulnaies, point de prêtre résidant ${ }^{67}$.

Ste-Anne-de-la-Pocatière, Pierre-Antoine Porlier, Canadien, âgé de 39 ans.

Rivière-Ouelle, Louis Chevalier, Canadien, âgé d'environ 64 ans.

Kamouraska, Joseph-Amable Trutaut, Canadien, âgé de 33 ans.

Rivière-du-Loup, point de prêtre résidant ${ }^{68}$.

L'Ile-Verte, point de prêtre résidant ${ }^{69}$.

Les Trois-Pistoles, point de prêtre résidant ${ }^{70}$.

Rimouski, le récollet Ambroise Rouillard, Canadien, âgé de 71 ans ${ }^{71}$.

\section{Gouvernement des Trois-Rivières}

Ste-Anne-de-la-Pérade, Louis-Michel Guay, Canadien, âgé de 42 ans.

Batiscan, le jésuite Charles Germain, Français, âgé de 57 ans ${ }^{72}$.

Ste-Geneviève-de-Bastiscan, point de prêtre résidant ${ }^{73}$.

Champlain, François Morrisseaux, Canadien, âgé de 42 ans.

Cap-de-la-Madeleine, point de prêtre résidant ${ }^{74}$.

65 Gareault quitte St-Roch-des-Aulnaies en novembre 1764; nommé curé au Cap-St-Ignace, il signe son premier acte le 20 novembre.

${ }_{66}^{6}$ La paroisse est desservie par le curé de l'Islet.

67 Depuis le départ de Gareault, la paroisse est desservie par le curé de Ste-Anne-de-la-Pocatière.

${ }^{68} \mathrm{La}$ paroisse est desservie par le curé de Kamouraska. Les premiers registres datent de 1813.

69 La paroisse paraît être desservie par le curé de Kamouraska. Les premiers registres sont commencés par le récollet Rouillard en 1766.

70 La paroisse est desservie par le récollet Rouillard.

71 Rimouski nous semble être le lieu de résidence de ce récollet; il signe avec le titre de « missionnaire de Rimousquy ».

72 Depuis le départ de Saint-Onge pour les Trois-Rivières, à l'automne de 1764 , c'est le jésuite Germain qui signe les actes.

${ }^{73} \mathrm{La}$ paroisse est desservie par le jésuite Germain; il y signe les actes à partir du 17 août 1764 .

${ }_{74}$ Le jésuite Germain y signe les actes jusqu'au 23 novembre 1764; entre cette date et le 13 juin 1765 , il y a un vide dans les registres; ce sera ensuite le chanoine Saint-Onge qui assurera la desserte. 
Les Trois-Rivières, le chanoine Pierre SaintOnge, Canadien, âgé de 42 ans ; grand-vicaire du Gouvernement des Trois-Rivières ${ }^{75}$; le récollet Hyacinthe Amiot, Canadien, âgé de 48 ans; Supérieur du Couvent et curé ;

le récollet Joseph Carpentier, Canadien, âgé de 37 ans $^{\mathbf{7 6}}$.

Les Forges, point de prêtre résidant ${ }^{77}$.

La Pointe-du-Lac, point de prêtre résidant ${ }^{78}$.

Yamachiche, Jacques-Maxime Chefdeville, Canadien, âgé de 50 ans.

Rivière-du-Loup [Louiseville], Médard Pétrimoulx, Canadien, âgé de 33 ans;

le récollet Dominique Pétrimoulx, Canadien, âgé de 29 ans ; frère du précédent.

Maskinongé, point de prêtre résidant ${ }^{79}$.

Yamaska, Joseph-Basile Parent, Canadien, âgé de 40 ans.

St-François-du-Lac, point de prêtre résidant ${ }^{80}$. Mission abénaquise, point de prêtre résidant ${ }^{81}$. Baie-du-Febvre, point de prêtre résidant $\mathbf{8 2}$.

Nicolet, Louis-Marie-Ferdinand Brassard, Canadien, âgé de 38 ans.

Bécancour, le récollet Louis Demers, Canadien, âgé de 32 ans ${ }^{83}$.

Mission abénaquise, point de prêtre résidant ${ }^{84}$.

\footnotetext{
${ }^{75}$ Nommé grand-vicaire pour succéder à Perrault, qui s'en va à Québec, Saint-Onge réside chez les Ursulines où il est aumônier.

${ }^{76} \mathrm{De}$ retour des Cèdres, il signe aux Trois-Rivières à partir du 29 janvier 1763.

${ }_{77}$ Cet endroit est desservi par les récollets des Trois-Rivières.

78 Depuis l'automne de 1763 , la paroisse est desservie par le curé d'Yamachiche.

${ }_{79} \mathrm{La}$ paroisse est desservie par les prêtres de la Rivière-du-Loup.

80 Le récollet Berey y signe son dernier acte le 27 juin 1763 , puis la desserte est assurée par le curé d'Yamaska; notons deux actes de Thiersant (le 7 août 1763) et un acte de Filiau, curé de Sorel (1er mars 1764 ); le récollet Berey reparaît le 20 janvier 1765 et signe avec le titre de «Miss. de chamblie ». Le reste du temps, c'est le curé d'Yamaska qui signe les actes.

81 Desservie par le curé d'Yamaska.

82 La paroisse est desservie par le curé de Nicolet.

83 Il y signe son premier acte le 27 août 1764 .

84 Les Abénaquis, n'ayant plus de chapelle, viennent à la paroisse.
} 
Gentilly, point de prêtre résidant ${ }^{85}$.

St-Pierre-les-Becquets, point de prêtre résidant ${ }^{86}$.

Gouvernement de Montréal

Berthier-en-haut, Louis-Marie-Melchior Kerberio, Français.

Lanoraie, le chanoine Joseph Gaillard, Canadien, âgé de 63 ans.

Lavaltrie, Basile Papin, Canadien, âgé de 42 ans.

Saint-Sulpice, le sulpicien Jean Matis, Français, âgé de 57 ans.

L'Assomption, le sulpicien Jacques Degeay, Français, âgé de 47 ans.

Repentigny, Philippe Daillebout Demusseaux, Canadien, âgé de 60 ans.

Lachenaie, Charles Duchouquet, Canadien, âgé de 58 ans ${ }^{87}$.

Mascouche, le jésuite Jean-Baptiste de la Brosse, Français, âgé de 40 ans ${ }^{88}$.

Terrebonne, Antoine Proulx, Canadien, âgé de 38 ans.

St-François-de-Sales, Pierre Marchand, Canadien, âgé de 32 ans.

St-Vincent-de-Paul, Ambroise Renoyer, Canadien, âgé de 44 ans.

Ste-Rose, François Petit, Canadien, âgé de 41 ans ${ }^{89}$.

La Pointe-aux-Trembles, le sulpicien Clément Pagés, Français, âgé de 49 ans.

Rivières-des-Prairies, le sulpicien Pierre Huet de la Valinière, Français, âgé de 32 ans.

La Longue-Pointe, le sulpicien Jean Beauzèle, Français, âgé de 41 ans.

85 Il n'y a pas encore d'église; la desserte est assurée par le curé de Champlain.

86 La paroisse est desservie par Hingan, curé de Deschaillons.

87 Il signe son dernier acte à St-Pierre-de-Montmagny le 15 septembre 1764 , et son premier acte à Lachenaie le 12 novembre 1764 .

88 Il se rend à l'occasion dans le Bas-de-Québec pour aider le P. Coquart, mais c'est à Mascouche qu'il réside; il y signe les registres jusqu'au 12 juin 1766.

89 Parti de Lotbinière à l'automne de 1761, il signe les actes de Ste-Rose à partir du 15 octobre 1761 . 
Sault-au-Récollet, le sulpicien Louis-Simon Pertuis, Français, âgé de 58 ans ${ }^{90}$.

Saint-Laurent, le sulpicien Jean-de-Dieu-François Robert, Français, âgé de 38 ans.

Montréal, le sulpicien Etienne Montgolfier, Français, âgé de 52 ans; il est encore Supérieur du Séminaire, mais il a démissionné comme grand-vicaire de Montréal;

le sulpicien Louis Jollivet, Français, âgé de 39 ans, curé de Montréal;

le sulpicien Michel Peigné, Français, âgé de 64 ans ;

le sulpicien Jean-François Defeligonde, Français, âgé de 37 ans;

le sulpicien Guillaume Chambon, Français, âgé de 55 ans;

le sulpicien Antoine Faucon, Français, âgé de 49 ans;

le sulpicien Gilbert-Alexis Favard, Français, âgé de 67 ans ;

le sulpicien Jean Gay, Français, âgé de 46 ans;

le sulpicien Claude Poncin, Français, âgé de 39 ans;

le sulpicien Henri Vallierre, Français, âgé de 59 ans;

le sulpicien Jean Brassier, Français, âgé de 35 ans;

le sulpicien Joseph-Marie Pontarion, Français, âgé de 41 ans; aliéné;

le séculier Amable-Simon Raizenne, Canadien d'ascendance anglaise, âgé de $\mathbf{4 5}$ ans ${ }^{91}$;

le jésuite Jean-Baptiste St-Pé, Français, âgé de 78 ans;

le jésuite Pierre-René Floquet, Français, âgé de 48 ans;

le jésuite Antoine Gordan, Français, âgé de 47 ans;

\footnotetext{
90 Il passe de la Pointe-Claire à Notre-Dame-de-Montréal, puis il signe les registres du Sault à partir du 30 juillet 1763 .

$91 \mathrm{Ce}$ malheureux Raizenne, adonné à l'ivrognerie, avait été retiré de la cure de Contrecoeur et placé à Notre-Dame-de-Montréal sous surveil. lance; il signe dans les registres à partir du 22 juillet 1764.
} 
le récollet Isidore Marsolet, Canadien, âgé de 45 ans, Supérieur du Couvent;

le récollet Bernardin de Gannes, Acadien, âgé de 60 ans;

le récollet Nicolas-Albert Couturier, Canadien, âgé de 59 ans.

Lachine, le sulpicien Pierre-Paul-François DeLagarde, Français, âgé de 35 ans ${ }^{92}$.

La Pointe-Claire, le sulpicien Jean-Baptiste Reverchon, Français, âgé de 37 ans ${ }^{93}$.

Ste-Anne-du-Bout-de-l'Ile, le sulpicien Pierre Sartelon, Français, âgé de 55 ans.

Ste-Geneviève, le sulpicien Jean-Pierre Besson, Français, âgé de 38 ans.

Oka, le sulpicien Jean-Claude Mathevet, Français, âgé de 47 ans; Supérieur depuis la mort de Guen ;

le sulpicien Francois-Auguste De Terlaye, Français, âgé de 40 ans;

le sulpicien Vincent-Fleury Guichart, Français, âgé de 35 ans.

Ile Perrot, point de prêtre résidant ${ }^{94}$.

Coteau-des-Cèdres, le récollet Elzéar Maugé, Canadien, âgé de 43 ans ${ }^{95}$.

Ile-Dupas, Mathieu Lataille, Canadien, âgé de 39 ans.

Sorel, Joseph-Hippolyte Filiau, Canadien, âgé de 30 ans.

St-Ours, Joseph-François Youville, Canadien, âgé de 40 ans.

Contrecœur, le sulpicien Jean-Baptiste Curatteau, Français, âgé de 35 ans ${ }^{96}$.

St-Denis, Jean-Baptiste Frichet, Canadien, âgé de 47 ans.

St-Antoine, Michel Gervaise, Canadien, âgé de d'environ 48 ans.

92 Il a remplacé Brassier à Lachine au début de 1763 .

93 Il y signe les registres depuis le 7 juin 1761 .

94 Cet endroit, où il y avait une chapelle, était probablement desservi par les sulpiciens.

95 Il signe les registres jusqu'au 6 novembre 1766 .

96 Il remplace le séculier Raizenne, retiré à Montréal; il signe les registres à partir du 26 mars 1764 . 
Verchères, Louis Mercereau, Canadien, âgé de 48 ans.

St-Charles, Henri-Marie Lataille, Canadien, âgé d'environ 53 ans.

Varennes, Antoine-Marie Morand, Canadien, âgé de 40 ans.

Boucherville, Etienne Marchand, Canadien, âgé de 57 ans; grand-vicaire du Gouvernement de Montréal 97.

Longueuil, Claude Carpentier, Canadien, âgé de 41 ans ${ }^{98}$.

St-Mathias, point de prêtre résidant ${ }^{99}$.

Chambly, le récollet Félix Berey, Canadien, âgé de 44 ans ${ }^{1}$.

Laprairie, Jacques Desligneris, Français, âgé de 62 ans.

Caughnawaga, le jésuite Joseph Huguet, Francais, âgé de 39 ans;

le jésuite Bernard Well, né en Europe, âgé de 40 ans.

Châteauguay, point de prêtre résidant ${ }^{2}$.

St-Constant, point de prêtre résidant ${ }^{3}$.

St-Philippe, Ignace Gamelin, Canadien, âgé de 33 ans.

La Présentation, mission abandonnée depuis 1760.

St-Régis, mission abandonnée temporairement.

\section{Région des Grands Lacs}

L'Assomption-du-Détroit, le jésuite Pierre Potier, Français, âgé de 56 ans.

Ste-Anne-du-Détroit, le récollet Simple Bocquet, Français, âgé de 61 ans.

Michillimakinac, le jésuite Pierre Du Jaunay, Français, âgé de 60 ans.

97 Depuis la démission de Montgolfier en septembre 1764.

98 C'est un séculier et non un récollet, comme nous l'avons démontré lors de notre inventaire de 1760 . Il signe les registres à partir du 23 août 1763. Chambly.

99 Desservi par le récollet Berey, qui fait les fonctions curiales à

1 Succédant au séculier Carpentier, le récollet Berey signe les registres à partir du 28 août 1763 .

2 Desservi par le jésuite Well.

3 Depuis la mort de Normanville, la paroisse est desservie par le curé de Laprairie. 


\section{Région des Illinois}

Cahokias (Ste-Famille), point de prêtre résidant ${ }^{4}$.

Fort de Chartres, le récollet Luc Collet, Français, âgé de 49 ans.

Prairie-du-Rocher, point de prêtre résidant ${ }^{5}$ St-Philippe, point de prêtre résidant ${ }^{6}$.

Ste-Geneviève, point de prêtre résidant ${ }^{7}$.

Kaskaskias, le jésuite Jean-Baptiste Aubert, Français, âgé de 42 ans;

le jésuite Sébastien Meurin, Français, âgé de 57 ans.

Vincennes, point de prêtre résidant ${ }^{8}$.

\section{Une grave pénurie de prêtres}

Ce clergé, qui a perdu près du tiers de ses effectifs en le bref intervalle de cinq ans, est plutôt clairsemé; certaines parties du pays sont même tout à fait dépourvues.

Dans l'immense région des Illinois, nous ne trouvons plus que 3 prêtres, et l'un d'eux, le jésuite Aubert, sera d'ailleurs obligé de partir très bientôt: il ne sera pas remplacé. Dans les Grands Lacs, l'état de l'Église est aussi triste: là aussi, il n'y a plus que 3 prêtres; dans quelques mois, il n'en restera plus que 2, car le missionnaire de Michillimakinac va quitter son poste et la mission va demeurer sans prêtre pendant quelques années. Dans ces conditions, le travail de l'évangélisation reste nul, la population française accaparant à elle seule la demi-douzaine de prêtres qu'elle a pu garder.

Le Gouvernement de Montréal est la plus favorisée de toutes les régions du pays. On trouve des prêtres dans tous les centres, sauf dans quatre: l'fle Perrot, Saint-Mathias, Saint-

4 Depuis la désertion du grand-vicaire Forget du Verger, la paroisse est desservie par le récollet Collet.

5 Desservi par le récollet Collet.

- Desservi par le même récollet.

7 On ignore s'il y eut desserte après l'expulsion du jésuite Lamorinie.

8 Le jésuite Devernai y signe son dernier acte le 24 . octobre 1763 et est ensuite expulsé du pays. Les registres ne recommencent que le 23 novembre 1765 , mais ils nous paraissent signer par un laĩque, Phillibert. 
Constant et Châteauguay; quatre centres dont la population nous paraît négligeable; d'ailleurs, même sous le régime français, les deux premiers (l'ile Perrot et Saint-Mathias) n'avaient pas de prêtre résidant.

Dans le Gouvernement des Trois-Rivières, la rareté des prêtres devient un problème très grave. Nous y avons énuméré 20 centres; or, dans 11 de ces centres, il n'y a pas de prêtre résidant: Sainte-Geneviève-de-Batiscan, Cap-de-la-Madeleine, les Forges, la Pointe-du-Lac, Maskinongé, Saint-François-du-Lac et sa mission abénaquise, la Baie-du-Febvre, la mission abénaquise de Bécancour, Gentilly et Saint-Pierre-les-Becquets. Si deux ou trois de ces centres comptent une faible population, d'autres, cependant, sont des paroisses normalement peuplées et capables de faire vivre un prêtre. Dans un même secteur, nous trouvons trois centres importants qui n'ont point de prêtres: Saint-François-du-Lac, la mission abénaquise et la Baie-du-Febvre. Le vide ici s'explique par l'exil que le gouverneur Burton a imposé au récollet Berey: le récollet a été obligé de quitter Saint-Françoisdu-Lac sans trouver personne pour le remplacer. Et c'est ainsi que le curé d'Yamaska, qui est déjà à la tête d'une paroisse populeuse, doit desservir, en outre, Saint-François-du-Lac et la mission abénaquise. Sur cette même rive sud, près de la frontière orientale du Gouvernement, point de prêtre dans trois centres qui se suivent: la mission abénaquise de Bécancour, Gentilly et Saint-Pierre-les-Becquets.

Il s'est fait des vides dans le Gouvernement de Québec et ces vides n'ont pas été remplis. En ne tenant compte que des paroisses qui avaient leur prêtre avant la guerre, nous constatons que trois d'entre elles n'en ont plus: Saint-Jean de l'île d'Orléans, les Grondines et Saint-Roch-des-Aulnaies. Certains prêtres ont fort à faire: le curé Chaumont s'occupe de la BaieSaint-Paul, de l'lle-aux-Coudres et de la Petite-Rivière-SaintFrançois; le récollet Loiseau dessert les trois centres de la Beauce; le curé de Kamouraska doit voyager jusqu'à l'île-Verte; le jésuite Coquart doit couvrir tout le pays qui s'étend depuis les Sept-Iles jusqu'aux Éboulements; des Trois-Pistoles jusqu'à l'ex- 
trémité orientale de la Gaspésie, il n'y a qu'un prêtre, le récollet Rouillard, et c'est un septuagénaire.

En Acadie, la situation est infiniment triste. Nous trouvons un seul prêtre catholique dans cette partie autrefois florissante par l'évangélisation: le récollet Bonaventure Carpentier, qui réside à Miramichy. Passé Miramichy, il n'y a plus de prêtre catholique: plus un seul prêtre à la rivière Saint-Jean, plus un seul dans l'île Saint-Jean, plus un seul dans l'Acadie péninsulaire et dans le Cap-Breton. Lorsque Pierre Maillard est mort au mois d'août 1762, n'ayant auprès de lui, paraît-il, qu'un ministre protestant qui lui rendit les derniers devoirs ${ }^{9}$, l'Église acadienne était morte elle aussi. L'Acadie était une dure leçon pour les catholiques du Canada; cette leçon leur apprenait qu'un clergé pouvait disparaître.

De fait, ce clergé semblait en voie de disparition: au début de 1759 , il comptait 196 prêtres, c'est-à-dire un prêtre par 350 catholiques; à la fin de 1764, il n'a plus que 137 prêtres, soit un prêtre par 500 catholiques ${ }^{10}$; et cette chute va continuer de s'accélérer, au point qu'en 1790 il n'y aura plus qu'un seul prêtre par 1,400 catholiques.

\section{Les raisons d'espérer}

Puisque la séparation d'avec la France est devenue définitive et que cette séparation est accentuée par un ordre anglais qui interdit de faire venir des prêtres, il faut encore se demander si l'Église canadienne, en supposant qu'elle ait un évêque, peut assurer par elle-même son propre recrutement. En 1760 , les Canadiens et les Français se partageaient en deux groupes égaux, chacun représentant une proportion de $50 \%$. Depuis, cette proportion s'est modifiée: $55.9 \%$ du clergé est Canadien, et le reste est composé de Français de France. C'est là seulement une

9 Burns, The Abbé Maillard and Halifax, dans Soc. can. hist. Egl., rapport 1936-37, II : 22.

10 Nous basons notre calcul sur une population de 70,000 âmes. C'est ici une simple approximation; les recensements précis n'existent pas sous le régime militaire: il y a bien les recensements de 1762 et de 1765 , mais ils ne nous donnent pas la population des villes de Québec et de Montréal. 
moyenne très générale; l'étude de chacun des groupes est plus intéressante :

Prêtres séculiers ${ }^{11}$
Récollets
Messieurs de Saint-Sulpice
Jésuites
Messieurs du Séminaire
$\quad$ de Québec

\begin{tabular}{ccc} 
total & \multicolumn{3}{c}{ origine canadienne } \\
68 & 59 & $81.1 \%$ \\
22 & 17 & $73.9 \%$ \\
27 & 0 & $0.0 \%$ \\
16 & 0 & $0.0 \%$ \\
& & \\
4 & 0 & $0.0 \%$
\end{tabular}

Les prêtres séculiers proprement dits sont donc encore plus Canadiens qu'ils l'étaient en 1760: par les décès ou par les départs, le pourcentage canadien a passé de $82.1 \%$ à $88.1 \%$. En plus d'acquérir une plus grande homogénéité, ce clergé séculier répond parfaitement au vœu qu'exprimait le gouverneur Murray d'établir ici un clergé national; la protection de l'État lui est donc assurée. Surtout, le clergé séculier, habitué à se recruter chez les Canadiens, ne devrait pas avoir de difficulté à survivre, le jour où il aura un évêque. Autre détail qui confirme ce caractère de plus en plus canadien: la haute direction de l'Eglise du pays est confiée, à la fin de 1764, à trois Canadiens; en effet, les grands-vicaires Perrault, Saint-Onge et Marchand sont tous trois Canadiens. C'est en 1764 que ce phénomène se produit pour la première fois dans notre histoire.

Chez les Récollets, le pourcentage canadien, déjà élevé en 1760, n'a fait que s'accroître: de $70.8 \%$, il a monté à $73.9 \%$. Habitués à se recruter chez les Canadiens, les Récollets pourraient facilement se maintenir, mais ce point ne joue plus en leur faveur, puisqu'on leur refuse désormais de combler les vides qui se produiront.

Tout le reste du clergé est composé de Français de France: les 27 sulpiciens, les 16 jésuites et les 4 Messieurs du Séminaire de Québec. Ces trois Communautés auront à faire face au problème du recrutement canadien. Pour le Séminaire de Québec, ce problème, à vrai dire, n'en sera pas un, puisque, depuis un

11 Des 68 prêtres séculiers, l'origine d'un seul nous est inconnue. (celle de Chaumont, curé de la Baie-Saint-Paul): ce prêtre n'entre pas dans le compte de cette moyenne. 
siècle, c'est au Séminaire de Québec que les séculiers vont se former; les Canadiens y sont donc chez eux. Pour les Sulpiciens, le problème devrait être difficile, puisque les premiers Canadiens qui y entreront, vont se trouver dans une maison tout à fait étrangère: de fait, le problème sera très difficile, l'assimilation de cette Communauté par les Canadiens deviendra un véritable drame au début du dix-neuvième siècle et ce drame mettra en péril la Communauté elle-même. Quant aux Jésuites, le problème ne se pose pas, puisqu'on leur interdit de se recruter.

Cette Église, qui dans l'ensemble devient de plus en plus canadienne, trouve un autre avantage dans sa jeunesse relative. Son âge moyen est de 46.3 ans à la fin de 1764 . Elle n'a donc en quatre ans vieilli que d'environ 2 ans, puisqu'elle avait 44.5 ans en 1760. Ce sont les séculiers qui forment le groupe le plus jeune:

Prêtres séculiers ${ }^{12}$ Messieurs de Saint-Sulpice Récollets Jésuites

$\begin{array}{cc}\text { nombre } & \text { âge moyen } \\ 68 & 44.1 \\ 27 & 45.4 \\ 22 & 50.3 \\ 16 & 52.7\end{array}$

Nous ne parlons pas ici des Messieurs du Séminaire de Québec: il nous manque l'âge de l'un d'entre eux; or, comme ils ne sont que quatre, cet âge est essentiel si l'on veut trouver une moyenne qui soit vraiment sûre ${ }^{13}$.

Moins on est nombreux, plus on est vieux. Et il se trouve ici que les plus âgées des Communautés d'hommes sont celles qui n'oxt plus la permission de se recruter. Les Jésuites ont une moyenne d'âge de 52.7 ans; deux vieillards contribuent à tenir cette moyenne élevée: le $\mathrm{P}$. Richer a 82 ans, et le P. St-Pé en a 78. L'âge des plus jeunes ne peut suffire à corriger cette situation, parce que les plus jeunes avancent déjà en âge: le benjamin (c'est le P. Huguet) a 39 ans. Mgr Briand recourra à un stra-

\footnotetext{
12 Nous ignorons l'âge de Kerberio, mais notre moyenne est établie sur une base de 67 .

${ }^{13}$ L'âge du supérieur Boiret nous est inconnu; Pressart a 41 ans; Gravé, 34 ; Récher, 40.
} 
tagème en ordonnant prêtres deux Frères qui avaient 36 ans en 1764: les Frères Casot et Noël; et un autre, le Frère Maquet, âgé de 54 ans. Les deux premiers pouvaient assurément prolonger la vie du groupe.

Les Récollets sont dans une meilleure position. Le religieux qui contribue à élever la moyenne d'âge, c'est le P. Quintal, qui a 81 ans; mais les Récollets comptent 5 prêtres qui n'ont pas 40 ans. Trois sont très jeunes: le $P$. Demers, 32 ans; le $P$. Veyssière, 30 ans; et le P. Pétrimoulx, 29 ans. Comme ces derniers ont des chances de vivre encore pendant 35 ou 40 ans, on peut espérer que, dans l'intervalle, le Gouvernement anglais laisse tomber sa proscription.

Chez les Sulpiciens, il n'y a pas de grands vieillards; le plus âgé (Favard) a 67 ans: il est donc beaucoup moins vieux que le doyen des Jésuites et que celui des Récollets. Les Sulpiciens n'ont aucun octogénaire, aucun septuagénaire et seulement deux prêtres qui soient dans la soixantaine. De plus, ils comptent 11 prêtres sur 27 qui n'ont pas encore 40 ans; le benjamin est Huet de la Valinière, il n'a que 32 ans. Cette réserve de jeunesse expliquera pourquoi les Sulpiciens tarderont tant à s'agréger des Canadiens ${ }^{14}$; ils pourront attendre longtemps, sans trop d'inquiétude, que l'Angleterre leur permette de s'agréger des Sulpiciens de France.

On s'attendrait à trouver bien des vieillards chez les prêtres séculiers, puisque ces séculiers sont au nombre de 68. Or ils sont rares: le doyen des séculiers, Ménage, a 80 ans; après lui, nous ne trouvons aucun septuagénaire. Six prêtres seulement, sur 68, sont dans la soixantaine ${ }^{15}$. Il y a plus que cela: le tiers de ce clergé séculier n'a pas 40 ans! Le benjamin, Pierre Mennard, n'a que 25 ans. C'est là pour l'Église canadienne un grand réconfort: même si la solution au problème épiscopal tarde à venir, le clergé séculier peut encore attendre. Il y a des prêtres pour

${ }^{14}$ Le premier à s'agréger sera Pierre-Mathieu Gamelin-Maugras en 1767, mais il faut ensuite attendre en 1773 pour noter l'agrégation d'un second Canadien, François-Xavier Dézéry.

${ }_{15}$ Ce sont Louis Chaumont, 64 ans; Louis Chevalier, 64 ans; Philippe Daillebout Demusseaux, 60 ans; Jacques Desligneris, 62 ans; JosephAmbroise Gaillard, chanoine, 63 ans; Joseph Resche, chanoine, 69 ans. 
longtemps encore; la messe s'est éteinte dans l'Acadie péninsulaire, dans le Cap-Breton et dans l'île Saint-Jean, mais, quel que soit le retard qu'on apporte au règlement de la question religieuse, la messe pourra se dire pendant bien des années encore dans la vallée du Saint-Laurent.

MARCEL TRUDEL,

Professeur titulaire d'Histoire du Canada à l'Université Laval.

N.B. - Invitation pressante, à chacun de nos abonnés, de nous trouver un nouvel abonnement. 